\title{
CONTROLLING PROPERTIES IN Fe2O3 NANORODS OBTAINED BY SUCROSE
}

\author{
Jesus, J. R. ${ }^{1}$; Lima, R.J.S. ${ }^{2 *}$; Duque, J.G.S. ${ }^{1}$; Meneses, C.T. ${ }^{1}$ \\ ${ }^{1}$ Departament of Physics, Federal University of Sergipe, Itabaiana, Sergipe, Brazil \\ ${ }^{2}$ Academic Unit of Physiscs, Federal University of Campina Grande, Campina Grande, Paraíba, Brazil \\ *rodrigo@df.ufcg.edu.br
}

In the last years several methods to obtain nanostructured materials has been developed to control the size, distribution size and the shape. Therefore, these methods use precursors expensive materials or they are enough difficult. In this work, we have studied the role of the addition of sucrose on the structural and magnetic properties of $\alpha-\mathrm{Fe}_{2} \mathrm{O}_{3}$ nanoparticles obtained by coprecipitation method. The precursors have been prepared for adding different concentrations of the sucrose as chelating agent. To obtain the nanoparticles these precursors were heated in the temperature range between 200 and $400^{\circ} \mathrm{C}$. Samples have been characterized via X-ray diffraction (XRD), scanning electron microscopy (SEM), transmission electron microscopy (TEM) and magnetization measurements. $\mathrm{XRD}$ data confirm that crystalline phase is already formed at temperatures around $200^{\circ} \mathrm{C}$ showing a preferential growth to (110) crystallographic plane to the sample at $0.01 \mathrm{~mol} / \mathrm{L}$ of sucrose. These results have been confirmed by TEM images in which show that the nanorods present mean size of $20 \mathrm{~nm} x 44 \mathrm{~nm}$. M vs H measurements show a superparamagnetic behavior at room temperature. Analysis performed in the Zero Field Cooling and Field Cooling (ZFC-FC) magnetization data show clearly the dependence of the size, shape and size distribution of the samples as function of the chelating agent concentration and synthesis temperature. Besides, the samples obtained at $400^{\circ} \mathrm{C}$ show Morin's transition indicating that particles present large size distribution. 